\title{
Current Interventional Management Strategies for Coronary Arteriovenous Fistulae
}

\author{
Awad Al-Qahtani, MD', Ayman Zakaria, MD², Ziyad M. Hijazi, MD, MPH, FACC, MSCAl ${ }^{3 *}$ \\ ${ }^{1}$ Department of Cardiology, Heart Hospital, Hamad Medical Corporation, Doha, Qatar \\ 2 Department of Radiology, Hamad Medical Corporation, Doha, Qatar \\ ${ }^{3}$ Sidra Heart Center, Sidra Medicine, Doha, Qatar, Qatar
}

\begin{abstract}
Coronary arteriovenous fistulae are uncommon abnormal connections between one of the coronary arteries and a heart chamber or another blood vessel, usually pulmonary vasculature or other venous vessels. Clinically significant fistulae may lead to ischemia of the segment of the myocardium perfused by the affected coronary artery. Therefore, closure of such fistulae is indicated. Transcatheter closure if feasible is recommended and can be achieved using different occlusion devices. This paper discusses the clinical classification of fistulae and the interventional approach to eliminate such fistulae with some case examples. The availability of new coils and catheters render the interventional approach safe and effective.
\end{abstract}

Copyright @ 2018 Science International Corp.

\section{Key Words}

Coronary fistula, Coil occlusion, Congenital heart disease, Interventional therapies

Coronary artery fistula is defined as an abnormal connection between one of the coronary arteries and a heart chamber or another blood vessel, usually pulmonary vasculature or other venous vessels. It is estimated to account for $0.2-0.4 \%$ of total congenital cardiac anomalies [1]. In 1908 Maude Abbott published the first pathological account of this condition [2]. (c) 2018 Journal of Structural Heart Disease Published by Science International Corp. ISN 2326-4004

Accessible online at: http://structuralheartdisease.org/
Bjork and Crafoord in 1947 performed the first successful surgical closure of a coronary fistula in a patient with a preoperative diagnosis of patent ductus arteriosus [3].

In general, most coronary artery fistulae are small and do not cause any symptoms. Most are clinically undetectable and are found incidentally on echocardiography performed for other reasons or in adults undergoing coronary angiography performed for an unrelated cause. Most fistulae resolve spontaneously without causing any complications. Only those fistulae that are about three times the size of a normal caliber of a coronary artery may cause symptoms or complications and require management. Symptoms may include the following:

- Dyspnea on exertion

- Angina

- Fatigue

- Palpitations

Due to steel phenomenon, large fistulae may lead to ischemia of the segment of the myocardium perfused by the affected coronary artery. The mechanism is related to the diastolic pressure gradient and runoff from the coronary vasculature to a low-pressure receiving cavity/vessel. If the fistula is large, the intracoronary diastolic perfusion pressure progressively diminishes.
* Corresponding Author:

Ziyad M. Hijazi, MD, MPH, FACC, MSCAI

Cardiac Program, Department of Pediatrics

Sidra Medicine

PO Box 26999, Doha, Qatar

Tel. +974 4003 6602; Fax: +974 4404 1779; E-Mail: zhijazi@me.com 
Prior to the era of echocardiography, the right coronary artery was considered to be the major site of origin of the fistulae (40-60\%), followed by the left anterior descending (30-60\%) then circumflex and a combination thereof. However, currently, we believe more fistulae originate in the left anterior descending artery. The right side of the heart (ventricle, pulmonary arteries, right atrium, coronary sinus, etc) is the major drainage (termination) site of most fistulae $(90 \%)[4,5]$.

Prior to 1990 's, surgical ligation was considered the treatment of choice with external ligation of the fistula preferred if possible. However, if the fistula is posteriorly located behind the heart, internal closure of the termination site on cardiopulmonary bypass offered a safe alternative [6]. Recurrence rate after surgical closure is about $10 \%$ [7].

Since the report by Reidy et al. [8], percutaneous closure in the cardiac catheterization laboratory has become the most common option for management of large fistulae. To enable successful and safe closure, it is imperative to define the anatomy of the fistula by selective coronary angiography. Detachable balloons, coils, devices, and vascular plugs all have been used successfully to close coronary fistulae $[9,10]$.

The purpose of this paper is to discuss the management decisions and details of transcatheter closure techniques.

\section{Management Decisions}

As mentioned above, small fistulae in an asymptomatic patient need not be closed. However, if the fistula is large or if the patient is symptomatic, closure is recommended. The decision process in managing fistulae depends on: site of origin of the fistula (proximal vs distal) [11], size of the fistula, patient's symptoms, presence of any complication caused by the fistula (angina, heart failure, endocarditis, rupture, etc), age of the patient, the anatomy of the fistula and presence of other indications to undergo an invasive procedure. The current recommendations by the AHA/ACC guidelines [12] include for Class 1: patients with continuous murmur should undergo exact delineation of the origin and termination of the fistula by either echocardiography or CT/MRI; patients with large fistulae should undergo closure (surgical or per- cutaneous) after delineation of the exact anatomy and finally, small-moderate fistulae with complications (ischemia, arrhythmias or ventricular dysfunction of unexplained etiology) should undergo closure. Last but not least, the approach of elimination of the fistula (surgical vs. transcatheter) depends on the expertise of the physicians involved in the management of the patient.

\section{Proximal Fistulae}

If small in size with no symptoms, observation is recommended and no medications. However, if the fistula is medium or large with or without symptoms, closure is recommended (surgical vs. transcatheter) followed by antiplatelets for at least one year.

\section{Distal Fistulae}

If small in size with no symptoms, observation is recommended with no medications. However, if medium in size with or without symptoms one has two options: closure followed by antiplatelets for one year or observation while receiving antiplatelets indefinitely. If the fistula is large with symptoms, closure is recommended, 6 hours post-closure, heparin should be started to keep PTT at 1.5 times normal while warfarin is started. Patients should be discharged home on Warfarin to keep INR around 2.5 for a period of 6-12 months [11]. Also, these patients should receive antiplatelets indefinitely. If the fistula is large with no symptoms, one has two options: either observation while receiving antiplatelets indefinitely or closure. If fistula is closed, one should treat as large fistula with symptoms.

Another important factor in the decision-making process is the size of the patient.

\section{Fistulae in small patients}

If small-moderate in size, they can be left alone until the patient is bigger. Spontaneous regression of fistulae has been reported [13], however, if the fistula is big and leading to cardiac symptoms, closure is recommended. Elective closure of moderate-large sized fistulae that are not causing symptoms is reasonable and can be performed once the child is an appropriate weight (approximately $>15 \mathrm{~kg}$ ).

If fistula is associated with other cardiac lesions (most commonly tetralogy of Fallot, patent ductus ar- 
teriosus, atrial septal defect), the fistula can be closed at the time of repair of the primary cardiac lesion.

\section{Transcatheter Closure Techniques}

Once a decision is made about the need for closure of the fistula, it is very important to plan the procedure appropriately. We recommend the following:

1. Discussion of the case with an interventional adult cardiologist colleague. We can't overemphasize the importance of collaboration with adult cardiologists when it comes to the coronary circulation. They need to be involved in the planning of the case.

2. Discussion of the case with an interventional radiologist to see what equipment may be needed. Interventional radiology uses many coils and catheters that are not readily available in the congenital cardiac catheterization laboratory. We have found over the years that getting help from interventional radiology has contributed to the success of the procedures.

3. If the patient is an adult age (over 18-21 years), we advise to admit the patient post-procedure into an adult unit equipped with continuous cardiac monitoring (telemetry) and with staff familiar with EKG's and management of cardiac ischemia. We believe this is an important aspect of managing such patients. The pediatric units, for the most part, are not familiar with cardiac ischemia/enzymes, and they may miss important events in these patients that could lead to catastrophe.

4. When placing coils/devices to occlude fistulae, it is very important to delineate the myocardial branches to ensure that coils/devices are beyond the last viable myocardial branch. Obviously, occlusion proximal to such branches will result in cardiac ischemia.

5. Available coils: coils are made of different materials, available in various lengths, diameters, shapes and several methods of delivery. Coils are available in lengths from 1 to $300 \mathrm{~mm}$ and in diameters ranging from 1 to $27 \mathrm{~mm}$. Available coil shapes include J- or C-shaped, helical, conical, tornado, straight, and complex three dimensional (3D) shapes. Coils may be bare or fibered with material such as Dacron, nylon fibers, polyester, wool, silk, or polyvinyl acetate (PVA) embedded within them to increase thrombogenicity. Steel was the initial material used for coils then came Platinum and different alloys that made them softer, more radio-opaque and non-ferromagnetic enabling future MRI follow-ups [14]. The methods of coil delivery had evolved over the last five decades in response to the need for a safer and more controllable deployment as well as to solve the technical problems encountered in old methods. Among the early methods were the pushable, injectable and liquid coils delivered by pushing wires or injecting saline or contrast after loading the coils in the delivery catheters [14]. The first detachable coil was described in 1977. Current detachable coils are deployed by a variety of mechanisms including mechanical, by electrolysis, and by hydrostatic means. The disadvantage of the mechanical detachment is that there is often friction between the coil and the microcatheter, during embolization through tortuous vessels, this friction can limit delivery, or the coil can rotate or flip at detachment [14]. Deployment of coils can be done by a wide range of catheters, the current assortment of microcatheters widely used may not be all well suited for several anatomic variants, including excessive vascular tortuosity. The most commonly used microcatheters for coiling are Excelsior SL-10 and 10-18 (Stryker, Kalamazoo, MI, USA), Echelon 10 and 14 (Medtronic, Minneapolis, MN, USA) and Headway 17 (Microvention TERUMO, Tustin, CA, USA). As mentioned above, these catheters are readily available in radiology departments engaged in aneurysm coiling. The proper coil for embolization should be sized 20 to $30 \%$ larger than what the target vessel measures on pre-deployment angiogram to prevent distal embolization or migration. Placement of an undersized coil risks its distal embolization away from the intended location. Attempting to place an oversized coil may result in the coil not forming the intended shape or even straightening in the vessel [14]. In general, dense packing of the target vessel is recommended to achieve complete embolization. The key to inducing complete thrombosis is cross-sectional occlusion which can be done by different techniques [15]. A scaffold technique involves initially deploying a higher radial force coil followed by a 
softer coil or an anchoring technique where a distal coil is anchored in a branch vessel then packed proximally [15]. This prevents distal migration and results in a tighter coil pack. An inflated compliant occlusion balloon may be used distal to the coil delivery catheter to prevent unwanted distal embolization, especially in high-flow fistulae. Then the balloon can be deflated and withdrawn after the deployment of the first coil that acts as a future basket for further coils [14].

6. The Amplatzer family of devices have been used for percutaneous closure of fistulae. The muscular device, the Duct occlude and the vascular plugs ( $($, II, IV) all have been used successfully for the closure of these fistulae [16-20]. The advantage of the plugs over the conventional devices is the need for a smaller sheath/catheter for deployment, thus making retrograde delivery possible. For deployment of devices (muscular VSD device or the PDA device), perhaps the best approach is to form an arteriovenous wire loop and deployment of the device from the venous side (see example below). However, for the vascular plugs, it is possible to deploy them from the retrograde approach using the corresponding guide catheter or small delivery sheath.

7. Finally, we want to emphasize the importance of anticoagulation and antiplatelet therapy post device/coil closure of fistulae. As discussed above [11], in some patients intravenous heparin has to be initiated about six hours after closure and bridging to Warfarin and antiplatelet therapy thereafter. This is extremely important to avoid the unfortunate complication of thrombus propagation proximal to the devices/coils [21].

\section{Techniques}

Fistulae can be closed either in a retrograde fashion (approach from the arterial system) or from the venous side (direct access if possible or after establishing an arteriovenous wire loop). Each technique has its own merits.

\section{Retrograde approach}

Access should be obtained via the right femoral artery and vein. We usually insert a 4-5Fr sheath in the artery in children and $6 \mathrm{Fr}$ in adults. For the vein, a cor- responding size can be used. On occasions, we also obtain access in the contralateral femoral artery. We do this if we use a 4-5Fr diagnostic coronary catheter for closure. The purpose of this is to perform control angiography for assessment of the position of coils/ devices prior to release. However, if we use a guide catheter, one may not need additional access. After a careful hemodynamic assessment is performed, selective coronary angiography is performed in the affected coronary artery. Usually, we perform at least two angiograms in different orthogonal views. The purpose of the angiograms is to delineate the exact anatomy of the fistula (origin, course, termination and viable myocardial branches). If the flow is brisk due to the size of fistula, one may need to balloon occlude the fistula with an end-hole balloon catheter advanced over a wire into the fistula and injection via this catheter after removal of the wire. We find this technique to be helpful in delineating the termination site and also in delineating the myocardial branches distal to the balloon (see case below).

\section{Case example:}

Four year young female child presented with continuous murmur heard shortly after birth. Echocardiography revealed the presence of moderate-large sized coronary artery fistula arising from the left anterior descending artery (LAD) and terminating in the right ventricle. She has been followed conservatively until age four years, when it was decided to close it on an elective basis. Her weight was $16.4 \mathrm{~kg}$. A 4Fr sheath was inserted in the right femoral artery and a $5 \mathrm{Fr}$ sheath in the right femoral vein. Selective left coronary angiography was performed using a 4Fr JL diagnostic catheter (Figure $1 \mathrm{~A})$. Then a $150 \mathrm{~cm} \times 6 \mathrm{~cm}$ Excelsior SL-10 Microcatheter (Stryker, Kalamazoo, MI, USA) was inserted inside the JL. The Transend floppy tip guidewire, 0.016" (Stryker, Kalamazoo, MI, USA) was used to navigate the tortuosity of the coronary artery until the wire reached to the right ventricle (Figure 1B). Then over this wire, a 4Fr balloon-tipped catheter was exchanged and positioned in the distal coronary artery. With balloon inflation, hand injection delineated the fistula better (Figure 1C). Then over the same wire, the balloon-tipped catheter was exchanged for the $\mathrm{JL}$ and then the Excelsior Microcatheter was fed over this wire all the way to the dis- 


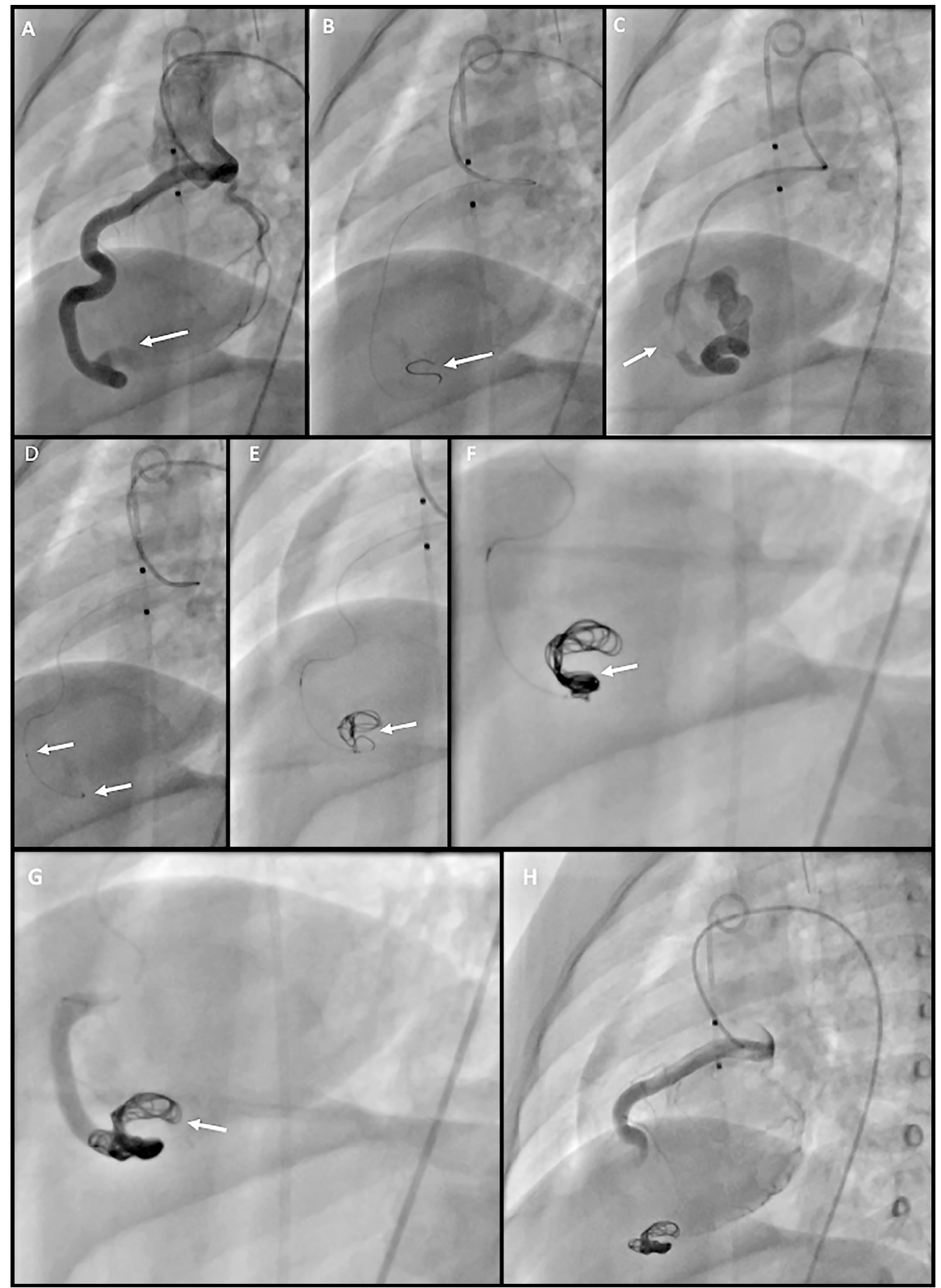

Figure 1. Selective Left Main Coronary Artery Angiograms in a 4 yr. young female child with left anterior descending (LAD) coronary artery to right ventricle (RV) fistula. Panel A. Dilated LAD terminating with a fistula to the RV (arrow). Panel B. Diagnostic JR catheter in left main and an 0.016"Transend guide wire all the way to RV (arrow). Panel C. A 4Fr. Balloon tipped catheter was advanced in LAD. Balloon inflated to block flow (arrow) and this delineated fistula better. Panel D. Cine fluoroscopy of the Excelsior Microcatheter in fistula. Two radio-opaque markers delineating position of the Microcatheter (arrows). Panel E. Cine fluoroscopy after deployment of first Target detachable coil (arrow) $8 \mathrm{~mm} \times 20 \mathrm{~cm}]$. Panel F. Cine fluoroscopy after 4 additional Target detachable coils were deployed (arrow) [second coil was also $8 \mathrm{~mm} \times 20 \mathrm{~cm}$, $3 \mathrm{rd}$ and 4 th coils were $4 \mathrm{~mm} \times 8 \mathrm{~cm}$ and last coil was $6 \mathrm{~mm} \times 20 \mathrm{~cm}$ ]. Panel G. Angiogram just after the deployment of the five coils revealed no flow distal to coil. Panel $\mathrm{H}$. Final angiogram shows flow stopped proximal to coils. 


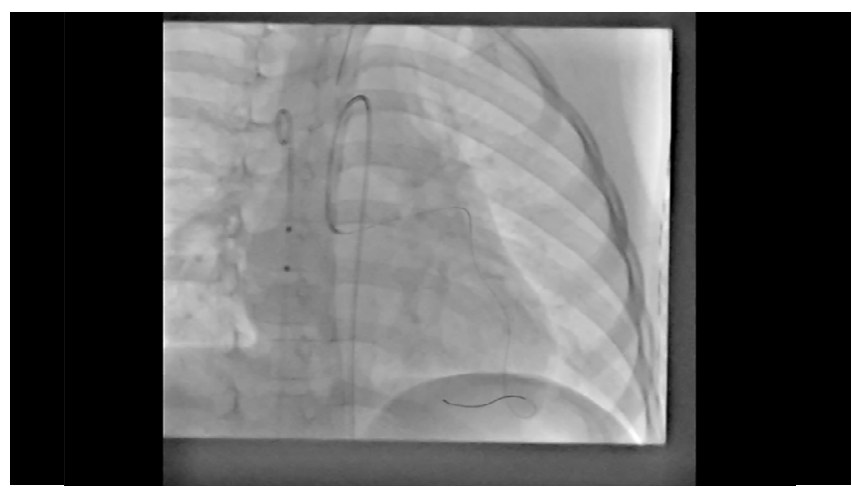

Video 1. Video which illustrates the case mentioned in figure 1. View supplemental video at https://doi.org/10.12945/j. jshd.2018.043.17.vid.01

tal part of the fistula (Figure 1D). The first coil used (Figure 1E) was $8 \mathrm{~mm} \times 20 \mathrm{~cm}$ Target detachable coil (Manufactured by Boston Scientific for Stryker Neurovascular). The advantage of such coils is its electrically released mechanism. Subsequently, four additional Target detachable coils were deployed (the second coil was also $8 \mathrm{~mm} \times 20 \mathrm{~cm}, 3^{\text {rd }}$ and $4^{\text {th }}$ coils were $4 \mathrm{mmx}-$ $8 \mathrm{~cm}$ and last coil was $6 \mathrm{~mm} \times 20 \mathrm{~cm}$ (Figure 1F). Repeat angiography between coil deployment was done to assess residual flow. Final angiogram after the fifth coil revealed good coils position and no residual flow (Figure 1G, H).

The patient was allowed to recover in the intensive care unit. After six hours and due to the sluggish coronary flow at the end of the procedure (Figure 1G, H), heparin drip was initiated at 15 units $/ \mathrm{kg} / \mathrm{hr}$ keeping PTT at 1.5 times normal. The same evening, the patient received $75 \mathrm{mg}$ aspirin and $2 \mathrm{mg}$ Warfarin. She was discharged home after 2 days on $75 \mathrm{mg}$ aspirin and $2 \mathrm{mg}$ Warfarin. For video of the case, see Video 1.

\section{Anterograde Approach}

As mentioned above, a closure can be done from the venous side either by creating an arteriovenous wire loop [16] or via direct access of the fistula from the venous side [22].

The wire loop technique: to do so, one has to cross the fistula from the arterial side and advance a wire until it exits into the right side and then snare and exteriorize from either the femoral vein or jugular vein depending on the location of the fistula.

\section{Case example:}

We previously have published this case [17]. 12 days young female baby, $2.4 \mathrm{~kg}$ presented in florid congestive heart failure due to a very large left main coronary (LMC) artery to the right ventricle fistula. Her right femoral artery was occluded due to a prior cardiac catheterization. Access was achieved from the left femoral artery $4 \mathrm{Fr}$, left femoral vein $4 \mathrm{Fr}$, and right internal jugular vein $8 \mathrm{Fr}$. The initial hemodynamic assessment revealed systemic pulmonary artery pressure and infinite Qp:Qs ratio. Angiography in the left main coronary artery revealed the presence of huge LAD to right ventricle fistula (Figure $2 \mathrm{~A}, \mathrm{~B}$ ). The fistula was crossed easily from the arterial side using a $0.035^{\prime \prime}$ floppy tip guidewire. The wire was advanced all the way to the main pulmonary artery and snared using a 4Fr., $10 \mathrm{~mm}$ gooseneck snare (Microvena) and was exteriorized from the right jugular vein (Figure 2C, D) creating an arteriovenous wire loop. An 8Fr. Mullins sheath was advanced over this wire from the jugular vein through the right ventricle into the fistula and into the distal LAD. The first device used was a $12 \mathrm{~mm}$ Amplatzer muscular VSD device (AGA Medical, Plymouth, MN)[Figure 2E). A total of 7 Flipper coils (five of them were $5 \mathrm{mmx} 8 \mathrm{~cm}$, and two were $5 \mathrm{mmx}-$ $10 \mathrm{cmm}$ (Cook Medical, Bloomington, IN) were deployed from the arterial side to create a nest behind the VSD device (Figure 2F, G). Repeat angiogram still revealed significant residual shunt (Figure $2 \mathrm{H}$ ). Due to the heavy contrast load used $(7 \mathrm{ml} / \mathrm{kg})$, the procedure was terminated. The baby remained stable without a rise in troponin or lactate but remained intubated with the continued moderate residual flow by echocardiography. Therefore, two days later the baby was brought back to the catheterization laboratory and a right carotid artery cut down was used and an $8 \mathrm{Fr}$. sheath was inserted. A 10/8 mm Amplatzer Duct Occlud (AGA Medical)(Figure 2l) and a $9 \mathrm{~mm}$ Gianturco Grifka Vascular Occlusion Device (Cook Medical)(Figure 2J) were deployed proximal to the coils and muscular VSD device. Repeat angiography revealed good devices positions and minimal residual flow (Figure $2 \mathrm{~K}, \mathrm{~L}$ ). Repeat hemodynamics revealed that the pulmonary artery pressure dropped to $40 \%$ systemic and the Qp:Qs ratio decreased to 2.7:1. The baby was extubated two days later. She was transferred back to referring institution on $4 \mathrm{mg} / \mathrm{kg}$ aspirin orally. She was 


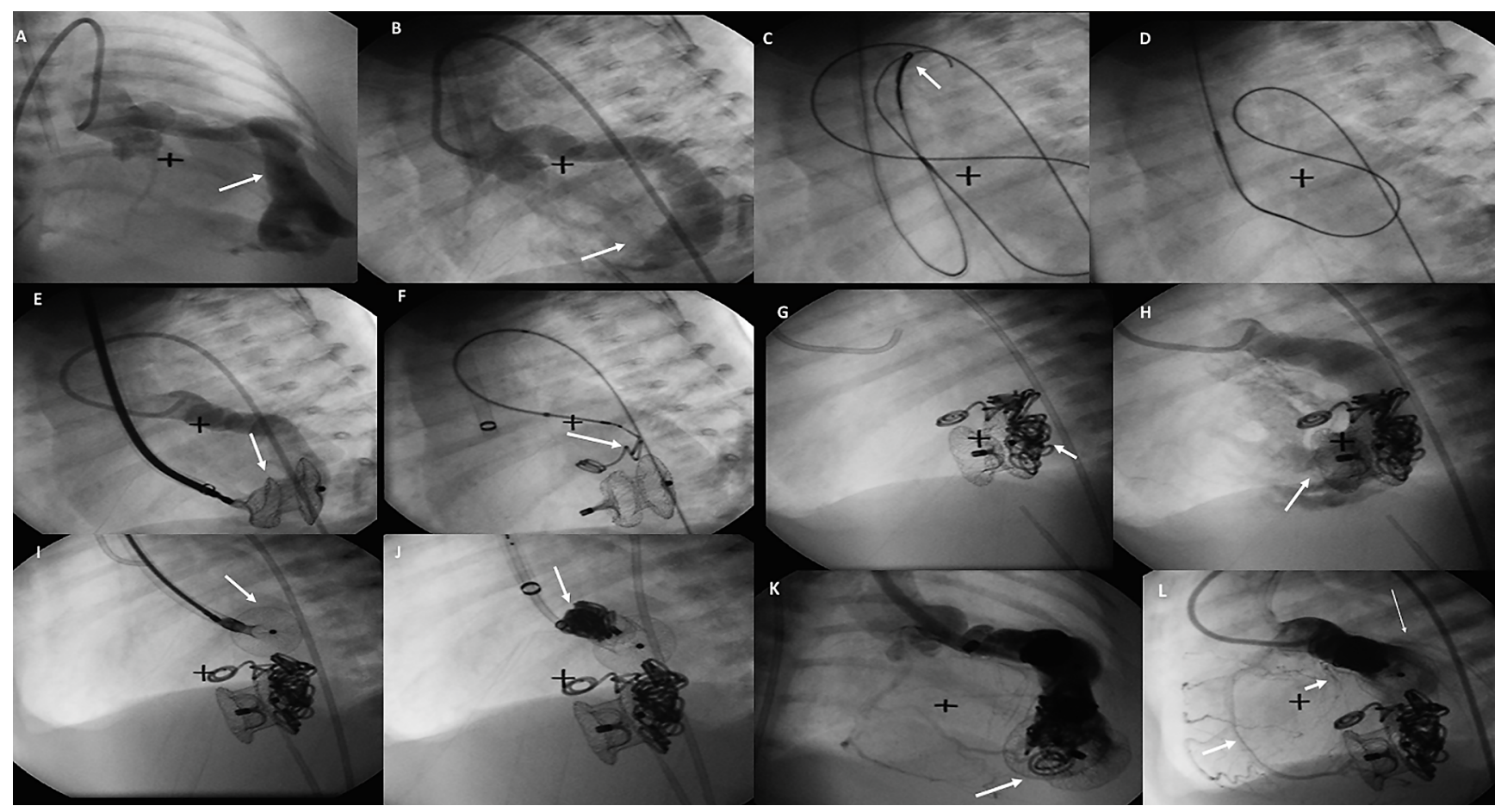

Figure 2. Selective left main coronary angiograms in a $2.4 \mathrm{~kg} 12$ days young baby with large left main coronary artery (LMC) fistula terminating in right ventricle (RV). Panel A. Angiogram in right anterior oblique (RAO) view showing large LMC artery fistula draining into RV. Panel B. Repeat angiogram in left anterior oblique (LAO) view showing drainage to RV (arrow). Panel C. Cine fluoroscopy after passing an $0.035^{\prime \prime}$ guide wire from fistula to RV to main pulmonary artery where it was snared using a gooseneck snare (arrow). Panel D. Forming of an arteriovenous wire loop with exit into the right internal jugular vein. Panel E. LMC artery angiogram after deployment of a $12 \mathrm{~mm}$ Amplatzer muscular VSD device (arrow) from the right internal jugular vein. Panel F. Cine fluoroscopy after deployment of 1st Flipper coil $(5 \mathrm{~mm} \times 8 \mathrm{~cm})$ from the arterial side (arrow) proximal to the device. Panel G. Cine fluoroscopy after deployment of additional 6 coils from the arterial side (arrow)[four of them were $5 \mathrm{~mm} \times 8 \mathrm{~cm}$ and two were $5 \mathrm{~mm} \times 10 \mathrm{cmm}$ ] to create a nest behind the VSD device. Panel H. Angiogram revealed residual flow through the fistula to the RV (arrow). Panel I. Cine fluoroscopy during deployment of a 10/8mm Amplatzer Duct Occlud (arrow) from right carotid artery cut-down proximal to the coils and VSD device. Panel J. Cine fluoroscopy during deployment of a $9 \mathrm{~mm}$ Gianturco Grifka Vascular Occlusion Device (GGVOD) (arrow) proximal to the PDA device. Panel K. Final angiogram in RAO view showing near complete occlusion of fistula (arrow). Panel L. Angiogram in LAO view showing devices with coils in good position with near complete occlusion. Thin arrow shows opacification of circumflex, fat short arrow shows LAD and fat long arrow shows right coronary artery.

discharged home after ten days from the procedure. She had been doing well since then. Echocardiography at one month revealed complete closure of the fistula and normal cardiac function.

\section{The direct access technique}

If the fistula could not be crossed from the arterial side to close retrogradely or to create wire loop, one may attempt to cross directly from the venous side (fistula exit) [22]. The following case illustrates this technique.

\section{Case Example:}

A 76-year-old gentleman was referred to us due to symptoms of increased shortness of breath. He was known to have a circumflex to pulmonary artery fistula for ten years prior to this procedure. He was a previous smoker. Pulmonary function test revealed a mild form of chronic obstructive pulmonary disease. Cardiac catheterization was performed via the right femoral artery using 7Fr. sheath and right femoral vein using 6 Fr. sheath. Hemodynamics revealed slightly elevated pulmonary artery pressure with a mean of $26 \mathrm{mmHg}$ and Qp:Qs ratio of 1.4:1. Angiogra- 


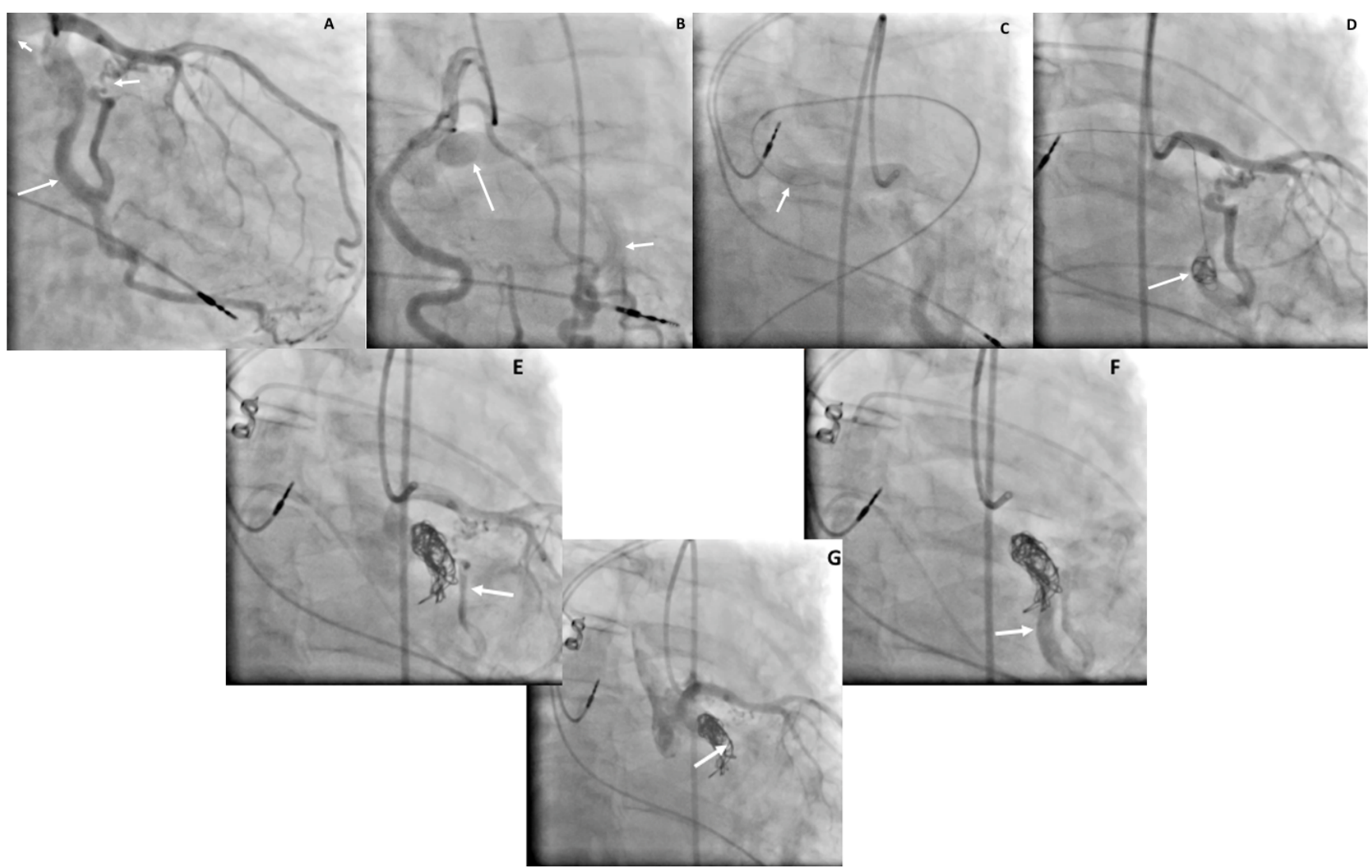

Figure 3. Panel $A$. Selective left main coronary angiogram in a 76 yr. old gentleman showing very narrow and tortuous circumflex (medium arrow). There is a fistula arising from circumflex (long arrow) and this fistula drained to the right pulmonary artery (short arrow). Panel B. Selective right coronary angiogram showing the posteromedial branch draining into a fistula (short arrow) that drains into the fistula that arose from the circumflex, all drain into the right pulmonary artery (long arrow). Panel C. Angiogram in LMC artery where fistula drains into mouth of right pulmonary artery, where a 5Fr. JR catheter was positioned (arrow). Panel D. Cine fluoroscopy during deployment of $12 \mathrm{~mm} \times 30 \mathrm{~cm}$ Target detachable coil (arrow) using the Excelsior Microcatheter. Panel E. angiogram after deployment of additional 7 Target detachable coils ( $2^{\text {nd }}$ coil: $10 \mathrm{~mm} \times 30 \mathrm{~cm} ; 3^{\text {rd }}$ and $4^{\text {th }}$ coils: $9 \mathrm{~mm} \times 20 \mathrm{~cm} ; 5^{\text {th }}, 6^{\text {th }}$ and $7^{\text {th }}$ coils: $4 \mathrm{~mm} \times 8 \mathrm{~cm}$ and $8^{\text {th }}$ coil: $3 \mathrm{~mm} \times 4 \mathrm{~cm}$ ) showing filling of the circumflex (arrow). Panel F. few frames later showing contrast up to the coils (arrow) and (Panel G), few frames later no residual flow in fistula and good coils position (arrow).

phy in the left main coronary artery revealed normal LMC and LAD. The circumflex origin was very narrow and very tortuous. At mid circumflex, a fistula arose and drained to the right pulmonary artery (RPA). The fistula size was double the size of the circumflex (Figure $3 \mathrm{~A}$ ). Angiography in the right coronary artery revealed normal artery with minimal coronary artery disease; however, the distal branch (posteromedial coronary artery) drained via smaller channels and connected with the circumflex fistula and all drained to the RPA (Figure 3B). Multiple attempts to cross the fistula from the LMC artery failed. Therefore, a 5Fr. JR catheter was used from the venous side to the RPA and crossed the exit site of the fistula (Figure $3 \mathrm{C}$ ). A $0.016^{\prime \prime}$ guidewire (Transend) was used to navigate the fistula. The wire and a $150 \mathrm{~cm} \times 6 \mathrm{~cm}$ Excelsior SL-10 Microcatheter were advanced all the way to the origin of the fistula from the circumflex. The wire was removed and a total of eight Target detachable coils were deployed in mid-distal fistula ( $1^{\text {st }}$ coil: $12 \mathrm{~mm} \times 30 \mathrm{~cm} ; 2^{\text {nd }}$ coil: $10 \mathrm{~mm} \times 30 \mathrm{~cm} ; 3^{\text {rd }}$ and $4^{\text {th }}$ coils: $9 \mathrm{~mm} \times 20 \mathrm{~cm} ; 5^{\text {th }}, 6^{\text {th }}$ and $7^{\text {th }}$ coils: $4 \mathrm{~mm} \times 8 \mathrm{~cm}$ and $8^{\text {th }}$ coil: $3 \mathrm{~mm} \times 4 \mathrm{~cm}$ (Figure 3D, E, F). Repeat angiography revealed complete closure of fistula (Figure 3G). The patient had recovered overnight and a repeat echocardiography the next day revealed complete closure of the fistula. He was discharged home after 24 hours from the procedure on his medications of warfarin and aspirin. 


\section{Follow-up}

It is mandatory to follow patients with coronary arteriovenous fistulae life-long. Such patients may be at increased risk for acute or late-onset coronary thrombosis [21-26], Patients with distal fistulae with dilated proximal conduit are especially at increased risk for such complications. We propose that such patients receive life-long antiplatelet therapy. The issue of anticoagulation needs to be taken into consideration as discussed above. Further, these patients need to undergo coronary imaging every few years based on symptoms or even periodic CT coronary angiography. Other factors may increase the risk of coronary thrombosis such as smoking, diabetes, hypertension, and hyperlipidemia. Patients with fistula drainage to the coronary sinus may be at higher risk of coronary thrombosis [27]. To best understand the long-term sequelae of coronary fistulae closure, there is an ongoing registry by the CCISC (Congenital Cardiovascular Interventional Study Consortium) collecting data on these patients.

\section{Conflict of Interest}

The authors have no conflict of interest relevant to this publication.

\section{Comment on this Article or Ask a Question}

\section{References}

1. Ogden JA. Congenital anomalies of the coronary arteries. Am J Cardiol. 1970;25(4):474-479. DOI: 10.1016/00029149(70)90016-0

2. Abbott M. Statistics of Congenital Cardiac Disease: 400 Cases Analyzed. J Med Res. 1908;19(1):77-81. PMID: 19971855

3. Björk G, Crafoord C. Arteriovenous aneurysm on the pulmonary artery simulating paten ductus arteriosus botalli. Thorax. 1947;2:65. DOI: 10.1136/thx.2.2.65

4. Tirilomis T, Aleksic I, Busch T, Zenker D, Ruschewski W, Dalichau H. Congenital coronary artery fistulas in adult: surgical treatment and outcome. Int J Cardiol. 2005;98(1):57-9. DOI: 10.1016/j.ijcard.2002.05.001

5. Ata Y, Turk T, Bicer M, Yalcin M, Ata F, Yavuz S. Coronary arteriovenous fistulas in the adults: natural history and management strategies. J Cardiothorac Surg. 2009;4:62. DOI: 10.1186/1749-8090-4-62

6. Albeyoglu S, Aldag M, Ciloglu U, Sargin M, Kemaloglu Oz T, Kutlu $\mathrm{H}$, et al. Coronary Arteriovenous Fistulas in Adult Patients: Surgical Management and Outcomes. Braz J Cardiovasc Surg. 2017;32(1):15-21. DOI: 10.21470/1678-9741-2017-0005

7. Said SA, Nijhuis RL, Op den Akker JW, Kimman GP, Van Houwelingen KG, Gerrits D, et al. Diagnostic and therapeutic approach of congenital solitary coronary artery fistulas in adults: Dutch case series and review of literature. Neth Heart J. 2011;19(4):183e191

8. Reidy JF, Sowton E, Ross DN. Transcatheter occlusion of coronary to bronchial anastomosis by detachable balloon combined with coronary angioplasty at same procedure. Br Heart J. 1983 Mar;49(3):284-7. DOI: 10.1136/hrt.49.3.284
9. Armsby LR, Keane JF, Sherwood MC, Forbess JM, Perry SB, Lock JE. Management of coronary artery fistulae. Patient selection and results of transcatheter closure. J Am Coll Cardiol. 2002;39(6):1026-1032. DOI: 10.1016/S0735-1097(02)01742-4

10. Qureshi SA. Coronary arterial fistulas. Orphanet J Rare Dis. 2006;1(51):51 (page 1-6)

11. Gowda ST, Forbes TJ, Singh H, Kovach JA, Prieto L, Latson LA, et al. Remodeling and thrombosis following closure of coronary artery fistula with review of management: large distal coronary artery fistula--to close or not to close? Cathet Cardiovasc Inte. 2013;82(1):132-42. PMID: 23408662

12. Warnes CA, Williams RG, Bashore TM, Child JS, Connolly HM, Dearani JA, et al. ACC/ AHA 2008 guidelines for the management of adults with congenital heart disease: Executive summary: A report of the American College of Cardiology/American Heart Association task force on practice guidelines. Circulation 2008, 2008;118:e714-e833

13. Hackett D, Hallidie-Smith KA. Spontaneous closure of a coronary artery-cardiac chamber fistula. Br Heart J. 1984;54:477-479. DOI: 10.1136/hrt.52.4.477

14. Vaidya S, Tozer KR, Chen J. An overview of Embolic Agents. Semin Intervent Radiol. 2008 Sep; 25(3):204-215. DOI: 10.1055/s0028-1085930

15. Osuga $\mathrm{K}$, Mikami K, Higashihara $\mathrm{H}$, Maeda $\mathrm{N}$, Tsuboyama T, Kuwabara M, et al. Principles and techniques of transcatheter embolotherapy for peripheral vascular lesions. Radiat Med. 2006;24(4):309-314. DOI: $10.1007 / \mathrm{s} 11604-006-2411-1$

16. Hakim F, Madani A, Goussous Y, Cao QL, Hijazi ZM. Transcatheter closure of a large coronary arteriovenous fistula using the new
Amplatzer $^{\mathrm{TM}}$ duct occluder. Cathet Cardiovasc Diagn. 1998;45:155-157. DOI: 10.1002/ (SICI) 1097-0304(199810)45:2<155::AIDCCD 11>3.0.CO;2-K

17. Holzer R, Waller III BR, Kahana M, Hijazi ZM. Percutanous closure of a giant coronary arteriovenous fistula using multiple devices in a 12 day old neonate. Cathet Cardiovasc Interv. 2003;60:291-294. DOI: 10.1002/ ccd. 10629

18. Regazzoli D, Giglio M, Besana F, Leone PP, Tanaka A, Ancona MB, et al. Multimodality evaluation of percutaneous closure of coronary fistula using Amplatzer Vascular Plug IV. Int J Cardiol. 2016;225:381-383. DOI: 10.1016/j.ijcard.2016.09.100

19. Li YF, Li YF, Xie ZF, Zhang X, Wang SS, Zhang ZW. Clinical application of Amplatzer Vascular Plug II in transcatheter closure of congenital coronary artery fistula in children. Zhonghua Er Ke Za Zhi. 2016;54(6):451455. PMID: 27256234

20. Bagul PK, Phadke MS, Kerkar PG. Percutaneous Closure of Left Main Coronary Artery Fistula Using Amplatzer Vascular Plug II and Amplatzer Duct Occluder I. JACC Cardiovasc Interv. 2016;9(7):e67-70. DOI: 10.1016/j.jcin.2015.12.279

21. Kharouf R, Cao QL, Hijazi ZM. Transcatheter closure of coronary artery fistula complicated by myocardial infarction. J Invasive Cardiol. 2007;19:E146-E149. PMID: 17470976

22. Wax DF, MaGee AG, Nykanen D, Benson LN. Coil embolization of a coronary artery to pulmonary artery fistula from an antegrade approach. Cathet Cardiovasc Diagn. 1997;42(1):68-69. DOI: 10.1002/ (SICI) 1097-0304(199709)42:1 <68::AIDCCD2O>3.0.CO;2-H 
23. Ascoop AK, Budts W. Percutaneous closure of a congenital coronary artery fistula complicated by an acute myocardial infarction. Acta Cardiol. 2004;59:67- 69. DOI: 10.2143/ AC.59.1.2005161

24. Hamada M, Kubo H, Matsuoka H, Kokubu $\mathrm{T}$, Oosuga $\mathrm{Y}$, Joh T. Myocardial infarction complicating surgical repair of left coronary-right ventricular fistula in an adult. Am J Cardiol. 1986;57:372-374. DOI: 10.1016/0002-9149(86)90935-5

25. Gowda ST, Latso LA, Kutty S, Prieto L. Intermediate to Long-Term Outcome Following Congenital Coronary Artery Fistulae Closure With Focus on Thrombus Forma- tion. Am J Cardiol. 2011;107:302-308. DOI: 10.1016/j.amjcard.2010.09.018

26. Toyoda $Y$, Yamaguchi M, Ohsima Y. A surgical case of right coronary artery-right atrial fistula complicated with postoperative myocardial infarction. Kyobu Geka. 1999;52:326 -331. PMID: 10226427

27. Valente AM, Lock JE, Gauvreau K, Rodriguez-Huertas E, Joyce C, Armsby L, et al. Predictors of Long-term adverse outcomes in patients with congenital coronary artery fistulae. Circ Cardiovasc Interv. 2010;3:134-139. DOI: 10.1161/CIRCINTERVENTIONS.109.883884
Cite this article as: Al-Qahtani $\mathrm{A}, \mathrm{Za}$ karia A, Hijazi ZM. Current Interventional Management Strategies for Coronary Arteriovenous Fistulae. Structural Heart Disease. 2018;4(5):212-221. DOI: https:// doi.org/10.12945/j.jshd.2018.043.17 